\title{
Evolving Treatment Patterns for Hospitalized COVID-19 Patients in the United States in April 2020-July 2020
}

This article was published in the following Dove Press journal: International Journal of General Medicine

\author{
Xiaozhou Fan (1D' \\ Barbara H Johnson (1D' \\ Stephen S Johnston' \\ Nivesh Elangovanraaj $\mathbb{D}^{2}$ \\ Paul Coplan (D) \\ Rahul Khanna (D) \\ 'Medical Device Epidemiology and Real \\ World Data Sciences, Johnson \& Johnson, \\ New Brunswick, NJ, USA; ${ }^{2}$ Mu Sigma Inc, \\ Bangalore, India
}

\begin{abstract}
We sought to examine the trend (April-July) in the treatment patterns among hospitalized COVID-19 patients using the Premier Healthcare Database (PHD). In the analysis, we identified 53,264 patients from 302 hospitalsthat continuously provided inpatient data from April 1, 2020 to July 31, 2020 to the PHD, a nationwide, population-based multihospital research database in the US. We used generalized estimating equations (GEE) models to assess changes in the proportion of therapies used during the study period. After adjusting for patient and provider factors, a decline in hydroxychloroquine and an increase in azithromycin and dexamethasone were observed among COVID-19 patients during the 4-month study period.
\end{abstract}

Keywords: COVID-19, hospitalization, treatment pattern

\section{Introduction}

Since emerging in the first quarter of this year, 17 million identified cases of SARSCoV-2 infection (commonly known as COVID-19) have been reported including more than 308,000 associated deaths in the US as of mid-December. The high hospitalization rate and lack of related experience and resources have caused unprecedented pressure on health systems to treat patients. Given the challenges, limited data are available to assess changes in the treatment of hospitalized COVID19 patients since the beginning of the pandemic in the US. In this study, we aim to evaluate the monthly treatment patterns of patients admitted to an inpatient setting with a diagnosis of COVID-19 in a large US multi-hospital database between April and July 2020.

\section{Method}

Patients with an inpatient admission between April 1, 2020 and July 31, 2020 with a COVID-19 diagnosis (International Statistical Classification of Diseases and Related Health Problems, Tenth Revision [ICD-10] code U07.1) were identified from 302 hospitals (118 [39.1\%] in South, 104 [34.4\%] in Midwest, 62 [20.5\%] in Northeast, 18 [6.0\%] in West), in the Premier Healthcare Database (PHD). To be included, hospitals were required to provide continuous inpatient data to the PHD during the study period. The PHD includes discharge-level information on patient demographics, diagnoses, procedures, discharge disposition, length of stay, and hospital and provider characteristics, among other features from more than 700 hospitals throughout the US. Although the
Correspondence: Xiaozhou Fan Medical Device Epidemiology and Real World Data Sciences, Johnson \& Johnson, 410 George St, New Brunswick, NJ 0890I, USA

Tel + I $908208-2625$

Fax + I 732 524-5242

Email xfan9@its.jnj.com 
database excludes federally funded hospitals (eg, Veterans Affairs), the hospitals included are nationally representative based on bed size, geographic region, location (urban/rural), and teaching hospital status. This study is exempt from Institutional Review Board (IRB) oversight because all patient-related data in the PHD is de-identified and HIPAAcompliant from both the inpatient and hospital-based outpatient settings (https://www.premierinc.com/newsroom/pressreleases/premier-healthcare-database-being-used-by-nationalinstitutes-of-health-to-evaluate-impact-of-covid-19-onpatients-across-the-u-s). Medications intended for antiviral therapy (hydroxychloroquine [HCQ] with or without azithromycin [AZM], remdesivir, lopinavir/ritonavir or other HIV protease inhibitors, ivermectin, convalescent plasma), therapy to treat the excessive immune response of the host in response to the viral infection (dexamethasone, methylprednisolone, tocilizumab, colchicine), antithrombotic therapy, and the angiotensin-converting-enzyme (ACE) inhibitor lisinopril were identified in the PHD hospital charge master records. Monthly trends (April-July 2020) in the proportion of patients treated with the selected therapies were examined. The relationships between admission month and therapies were assessed using generalized estimating equations (GEE) models ${ }^{1}$ accounting for potential clustering of outcomes within hospitals and adjusting for patient demographics, comorbidity status, and provider characteristics. GEE models instead of mixed effects models were applied to obtain the population average effect of covariates. Further, we conducted a subanalysis for patients admitted to the intensive care unit (ICU) to investigate if treatment administered to severe patients who need ICU care differs from treatment administered to general hospitalized patients. Analyses were performed using $\mathrm{R}$ software version 4.0.0.

\section{Results}

We identified 53,264 COVID-19 hospitalized patients, with 21,736 in April, 11,640 in May, 9,159 in June, and 10,729 in July. Patient and provider characteristics are shown in Supplementary Table 1. Proportions and adjusted odds ratios of therapies between the admission months are shown in Supplementary Tables and results for selected therapies are displayed in Figures 1 and 2. More than half of the patients used HCQ (32.6\% and 19.6\% with and without AZM, respectively) in April, followed by a steep decline to $<1 \%$ in July

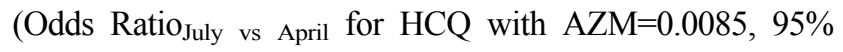
Confidence Interval $=0.0064-0.011$; OR July vs April for HCQ alone $=0.034,95 \% \mathrm{CI}=0.025-0.045$ ), while the use of AZM alone increased from $19.2 \%$ in April to $41.3 \%$ in July
$\left(\mathrm{OR}_{\text {July vs April }}=2.07,95 \% \mathrm{CI}=1.95-2.20\right)$. These trends are more notable in patients admitted to the ICU. A large increase in remdesivir use occurred between April and June, 13.1\% in overall patients $\left(\mathrm{OR}_{\mathrm{June}}\right.$ vs April $\left.=30.35,95 \% \mathrm{CI}=23.87-38.59\right)$, and $22.6 \%$ in patients admitted to the ICU in June $\left(\mathrm{OR}_{\text {June vs April }}=19.1,95 \% \mathrm{CI}=14.35-25.45\right)$ as compared to $0.4 \%$ and $1.2 \%$ in April, respectively; however, the use fell from June to July, $10.6 \%$ in overall patients $\left(\mathrm{OR}_{\text {July vs April }}\right.$ $=23.75,95 \% \mathrm{CI}=18.65-30.24)$ and $20.6 \%$ in ICU patients $\left(\mathrm{OR}_{\text {July vs April }}=16.53,95 \% \mathrm{CI}=12.32-22.18\right)$. Other antiviral drugs were used in $<2 \%$ of patients consistently from April to July. The largest percentage increase was observed for dexamethasone use (38-fold increase from $4.7 \%$ in April to $61.2 \%$ in July, $95 \% \mathrm{CI}=34.70-41.15$ ), but the use of another corticosteroid, methylprednisolone, decreased by $12 \%\left(\mathrm{OR}_{\text {July vs April }}\right.$ $=0.48,95 \% \mathrm{CI}=0.44-0.52$ ). No clear trend was observed in the use of other immunomodulators. Antithrombotic therapy was common and consistently used across the study period, $>85 \%$ overall and $>90 \%$ in ICU patients. The use of lisinopril increased slightly from $8.0 \%$ in April to $11.4 \%$ in July $\left(\mathrm{OR}_{\text {July vs April }}=1.36,95 \% \mathrm{CI}=1.24-1.49\right)$.

\section{Discussion}

In the absence of treatments approved by national regulatory authorities, providers have been navigating uncharted territory to manage and treat hospitalized COVID-19 patients. This is reflected in the variable trends we observed in therapies used among these patients after considering the differences in patient and provider characteristics in each month of the comparisons. Though widely used for COVID-19 treatment in the earliest stage of the pandemic, limited data support the efficacy of HCQ against COVID-19. A steep decline in HCQ use was observed in May forward, likely as a response to the April 24 safety alert followed by revocation of the emergency use authorization (EUA) by the FDA on June 15. Since then, more evidence has emerged indicating HCQ has no beneficial effects for COVID-19 patients. $^{2}$ AZM, commonly used as an adjunct to HCQ during the early phase of the pandemic, emerged as a solo therapy with increasing use because of its antimicrobial effects, especially among severe cases. We observed a substantial increase in the use of remdesivir from May to June followed by a slightly decline in July. The rapid growth in remdesivir use may be driven by the EUA issued on May 1, and several factors could contribute to the observed fluctuation in remdesivir use afterwards. Controversial data 


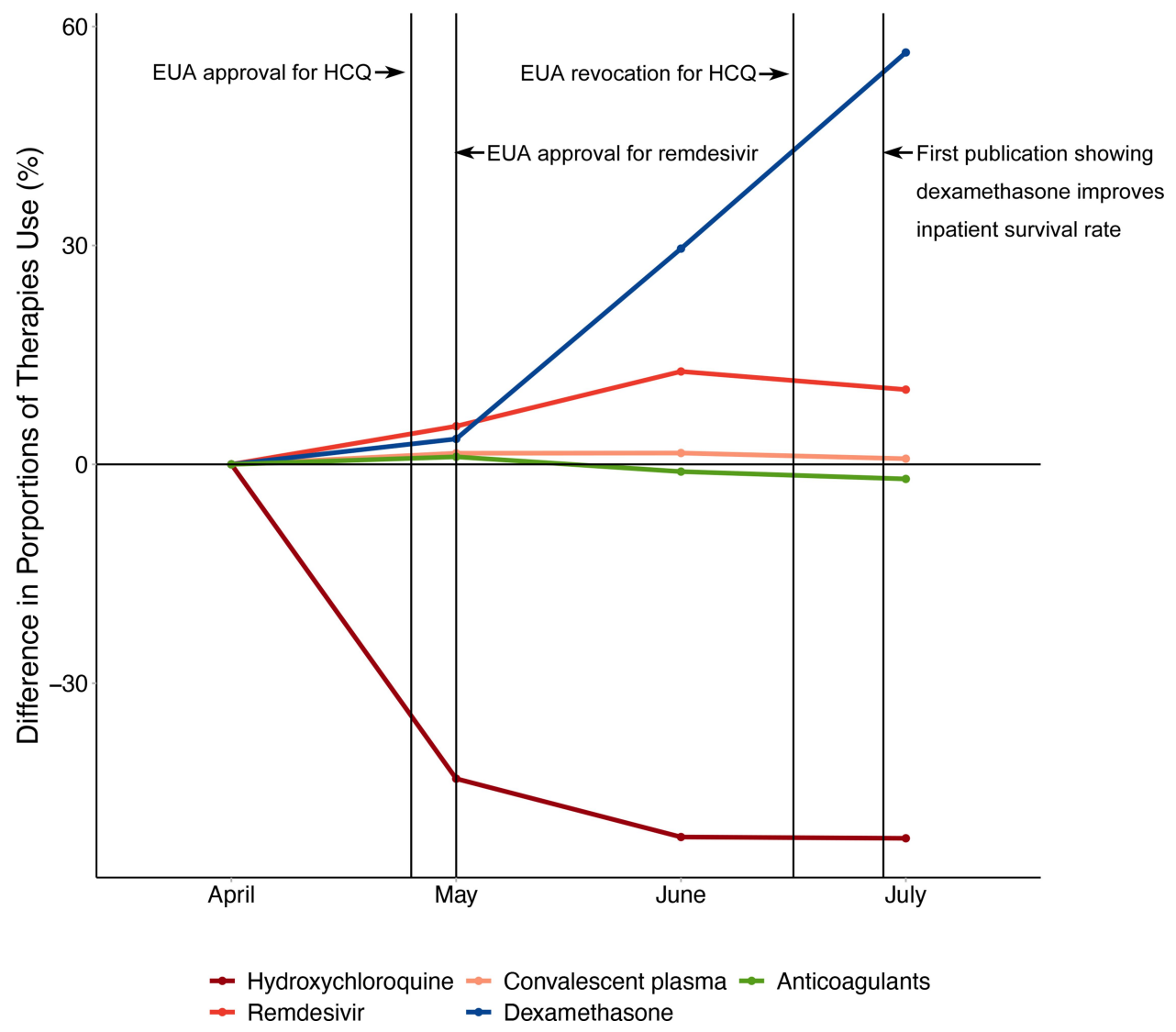

Figure I Changes in proportions of therapies use for COVID-19 from April to July in hospitalized patients in the US. Among 21,736 COVID- 19 hospitalized patients in April in our study, the proportions of therapies use were $52.2 \%$ for Hydroxychloroquine (HCQ) with and without Azithromycin (AZM), 0.4\% for remdesivir, I.6\% for convalescent plasma, $4.7 \%$ for dexamethasone, and $87.0 \%$ for anticoagulants. The line graph displays the differences in the proportions of these selected therapies use in May, June, or July compared to April. Proportions of therapies use in the overall study population and in patients admitted to the intensive care unit (ICU) from April to July are shown in Supplementary Tables 2 and $\underline{3}$.

regarding the efficacy and clinical importance of remdesivir, as one of the reasons, may cause decreased use of remdesivir around that time. ${ }^{3}$ Scientific evidence and National Institute of Health (NIH) treatment guidelines for COVID-19 regarding the benefit of corticosteroids for the hospitalized patients starts emerging after midJune. The effectiveness of dexamethasone in reducing mortality rate was demonstrated by the Randomized Evaluation of COVID-19 Therapy (RECOVERY) trial, a multicenter randomized trial in more than 6,000 hospitalized patients with COVID-19. ${ }^{4}$ In contrast, evidence to support the use of methylprednisolone for the treatment of COVID-19 is not as robust as that demonstrated in the RECOVERY trial. ${ }^{5}$ As such, dexamethasone is strongly recommended for hospitalized patients who require supplemental oxygen, and methylprednisolone is recommended if dexamethasone is not available. Thus, it should come as no surprise that dexamethasone use started increasing in June (from $8.2 \%$ in May to
$34.3 \%$ in June) and doubled in July (61.2\%), when it was preferentially used by doctors over methylprednisolone. In our study population, a consistently high proportion of patients received anticoagulants across the study period, consistent with the evidence indicating antithrombotic therapy is associated with a lower mortality rate among hospitalized patients who are at high risk of hypercoagulable states. ${ }^{6}$ Despite the biological plausibility that the use of ACE inhibitors increases susceptibility to COVID-19 and severity of the illness, ${ }^{7}$ we observed a mild trend increase in the use of lisinopril, which is often recommended for cardiovascular disease treatment but not for the treatment of COVID19. In conclusion, we observed that the trends in COVID-19 treatment in real-world data were reflective of the evolving scientific evidence, regulatory decisions, and federal guidelines. Although HCQ was widely used in April, either alone or in combination, with little evidence to support its use, fortunately its frequency of 


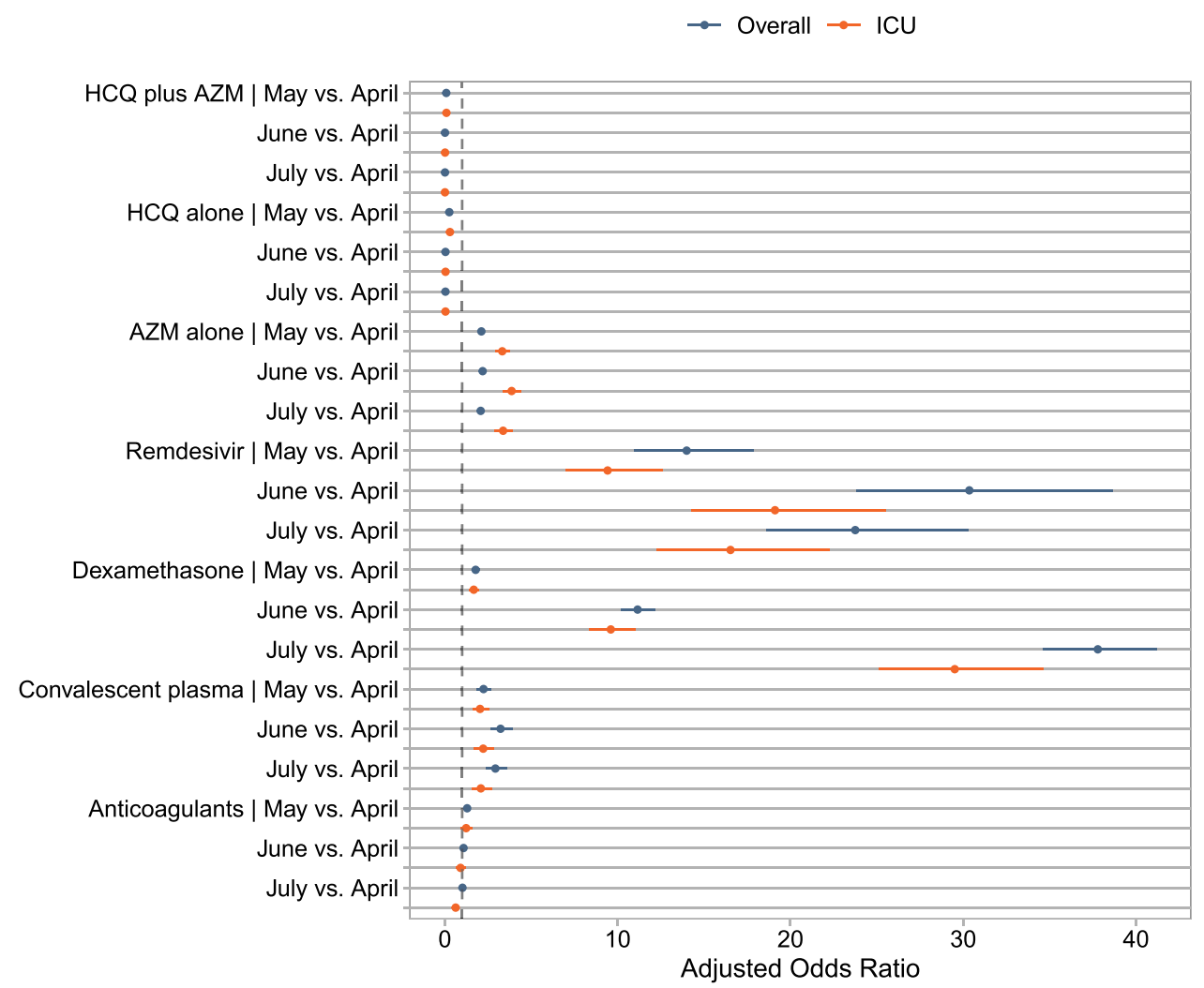

Figure 2 Adjusted odds ratios of therapies for COVID-19 in May, June, or July compared to April in hospitalized patients in the US. Multivariable regression using generalized estimating equations (GEE) with logit link and binomial distribution function were used to examine differences in the therapies use between the admission months. Patients and provider characteristics displayed in Supplementary Table I were adjusted for in the GEE model. The forest plot displays adjusted odds ratios and 95\% confidence intervals of therapies in May, June, and July compared to April in the overall study population and patients admitted to the ICU (see Supplementary Tables 4 and $\underline{5}$ ).

use rapidly decreased in May and June 2020 in response to evidence-based assessments in published journals and communications by the U.S. FDA. As better treatments for COVID-19 become available, continued monitoring and assessment of treatment patterns of hospitalized COVID-19 patients will be helpful.

\section{Acknowledgments}

Rusha Chaudhuri, Harikumaran R. Dwarakanathan, Aatheep Gurubaran, Joseph Guilfoyle, Pragya Rai

\section{Funding}

This study was sponsored by Johnson \& Johnson.

\section{Disclosure}

Xiaozhou Fan, Barbara H Johnson, Stephen S Johnston, Paul Coplan, and Rahul Khanna are employees of Johnson \& Johnson. Nivesh Elangovanraaj is an employee of $\mathrm{Mu}$ Sigma Inc. The authors report no other potential conflicts of interest in this work.

\section{References}

1. Ballinger GA. Using generalized estimating equations for longitudinal data analysis. Organ Res Methods. 2004;7(2):127-150. doi:10.1177/ 1094428104263672

2. Cortegiani A, Ippolito M, Ingoglia G, et al. Update I. A systematic review on the efficacy and safety of chloroquine/hydroxychloroquine for COVID-19. J Crit Care. 2020;59:176-190. doi:10.1016/j. jcrc.2020.06.019

3. McCreary EK, Angus DC. Efficacy of Remdesivir in COVID-19. JAMA. 2020;324(11):1041-1042. doi:10.1001/jama.2020.16337

4. Horby P, Lim WS, Emberson J, et al. Effect of dexamethasone in hospitalized patients with COVID-19: preliminary report. medRxiv. 2020. doi:10.1101/2020.06.22.20137273

5. Jeronimo CMP, Farias MEL, Val FFA, et al. Methylprednisolone as adjunctive therapy for patients hospitalized with coronavirus disease 2019 (COVID-19; Metcovid): a randomized, double-blind, Phase IIb, Placebo-controlled trial. Clin Infect Dis. 2020. doi:10.1093/cid/ ciaal177

6. Kollias A, Kyriakoulis KG, Dimakakos E, et al. Thromboembolic risk and anticoagulant therapy in COVID-19 patients: emerging evidence and call for action. Br J Haematol. 2020;189(5):846-847. doi:10.1111/ bjh. 16727

7. Saheb Sharif-Askari N, Saheb Sharif-Askari F, Al Heialy S, et al. Cardiovascular medications and regulation of COVID-19 receptors expression. Int J Cardiol Hypertens. 2020;6:100034. doi:10.1016/j. ijchy.2020.100034 
International Journal of General Medicine

Dovepress

\section{Publish your work in this journal}

The International Journal of General Medicine is an international, peer-reviewed open-access journal that focuses on general and internal medicine, pathogenesis, epidemiology, diagnosis, monitoring and treatment protocols. The journal is characterized by the rapid reporting of reviews, original research and clinical studies across all disease areas. The manuscript management system is completely online and includes a very quick and fair peer-review system, which is all easy to use. Visit http://www.dovepress.com/ testimonials.php to read real quotes from published authors.

Submit your manuscript here: https://www.dovepress.com/international-journal-of-general-medicine-journal 